\title{
Novel device-based interventional strategies for advanced heart failure
}

\author{
Gabor G. Toth ${ }^{1}$, Marc Vanderheyden², Jozef Bartunek² \\ ${ }^{1}$ Department of Cardiology, University Heart Centre Graz, Graz, Austria \\ ${ }^{2}$ Cardiovascular Research Centre Aalst, OLV Clinic, Aalst, Belgium
}

Adv Interv Cardiol 2016; 12, 1 (43): 13-16

DOI: $10.5114 /$ pwki.2016.56944

\begin{abstract}
A bstract
While heart failure is one of the leading causes of mortality and morbidity, our tools to provide ultimate treatment solutions are still limited. Recent developments in new devices are designed to fill this therapeutic gap. The scope of this review is to focus on two particular targets, namely (1) left ventricular geometric restoration and (2) atrial depressurization. (1) Reduction of the wall stress by shrinking the ventricular cavity has been traditionally attempted surgically. Recently, the Parachute device (CardioKinetix Inc., Menlo Park, CA, USA) has been introduced to restore ventricular geometry and cardiac mechanics. The intervention aims to partition distal dysfunctional segments that are non-contributory to the ventricular mechanics and forward cardiac output. (2) Diastolic heart failure is characterized by abnormal relaxation and chamber stiffness. The main therapeutic goal achieved should be the reduction of afterload and diastolic pressure load. Recently, new catheter-based approaches were proposed to reduce left atrial pressure and ventricular decompression: the InterAtrial Shunt Device (IASD ${ }^{T M}$ ) (Corvia Medical Inc., Tewksbury, MA, USA) and the V-Wave Shunt (V-Wave Ltd, Or Akiva, Israel). Both are designed to create a controlled atrial septal defect in symptomatic patients with heart failure. While the assist devices are aimed at end-stage heart failure, emerging device-based percutaneous or minimal invasive techniques comprise a wide spectrum of innovative concepts that target ventricular remodeling, cardiac contractility or neuro-humoral modulation. The clinical adoption is in the early stages of the initial feasibility and safety studies, and clinical evidence needs to be gathered in appropriately designed clinical trials.
\end{abstract}

Key words: heart failure, pediatric interventional cardiology, novel technologies.

\section{Introduction}

While heart failure is one of the leading causes of mortality and morbidity, being associated with increasing socio-economic costs, our tools to provide ultimate treatment solutions are still limited. The hallmark of heart failure is the loss of myocytes, leading to replacement fibrosis, increased hemodynamic stress, activation of deleterious neuro-humoral, inflammatory signaling and increased oxidative stress. The reduced cardiac performance and increased wall stress perpetuate the reactive molecular and tissue signature, resulting in maladaptive remodeling characterized by increased ventricular mass and composition with altered volume and geometry. As the left ventricle becomes less elliptical and ventricular sphericity increases, these changes in shape contribute to the reduction in radial, circumferential and longitudinal strains.

Given the causal relationship between remodeling and reduced pump function, therapies that decrease the elevated myocardial stress are the cornerstone in the management of heart failure. Such therapies are aimed to induce the so-called 'reverse remodeling'. From the pathophysiological view, the term 'reverse remodeling' has been adopted to reflect the beneficial effects that induce a leftward shift in the ventricular pressure-volume relationship in parallel with ventricular size reduction. Multimodal treatments, including life-style management, pharmacological therapies and various device-based interventions, have been proposed to induce reverse remodeling and are indisputably associated with improvements in morbidity and mortality in defined subsets of heart failure patients. Yet, despite these undisputable advances, morbidity and mortality remain high in advanced heart failure, with a persisting therapeutic gap in this patient population. Recent developments in new devices are aimed at filling this therapeutic gap. The scope of this review is to focus on two particular targets, name-

\section{Corresponding author:}

Gabor G. Toth MD, Department of Cardiology, University Heart Centre Graz, Auenbruggerplatz 15, 8036 Graz, Austria, phone: +436606376479 , e-mail: gabor.g.toth@medunigraz.at

Received: 9.12.2015, accepted: 1.01.2016. 
ly (1) left ventricular geometric restoration in systolic heart failure and (2) atrial depressurization in diastolic heart failure.

\section{Left ventricular geometric restoration}

Left ventricular enlargement serves initially to sustain the stroke volume and ventricular function. However, as outlined above, in the long term, it is associated with increased wall stress, precipitating a vicious cycle of progressive worsening and decline in cardiac performance. Specific subsets of patients prone to this development are those with extensive anterior myocardial infarction. Here, the extensive damage with thinning of the anterior and apical segments favors scar expansion, ventricular elongation and dilation. In accordance with Laplace's law, the altered ventricular geometry increases regional wall stress, and this self-perpetuating cycle deleteriously affects spherical chamber remodeling. In addition, apical wall motion abnormalities compromise the cardiac mechanics and efficiency.

Reduction of the wall stress by shrinking the ventricular cavity has been traditionally attempted surgically. Recently, the Parachute device (CardioKinetix Inc., Menlo Park, CA, USA) has been introduced to restore ventricular geometry and cardiac mechanics. The intervention aims to partition distal dysfunctional segments that are non-contributory to the ventricular mechanics and forward cardiac output.
The partitioning is achieved using a parachute-shaped device excluding dysfunctional apical segments from mid and basal segments with preserved contractile function (Figure $1 \mathrm{~A}-\mathrm{B}$ ). In other words, the Parachute device partitions the enlarged ventricle into a "dynamic" and a "static" chamber, where the latter becomes practically excluded from the circulation, normalizing the physiologic conical shape of the dynamic chamber, as well.

The device consists of an umbrella-like flexible nitinol frame, covered with poly-tetra-fluoro-ethylene surface. The nitinol frame provides a stable and well-expanded position below the papillary muscles, leaning against the apex. The flexible frame allows the device to follow the contractions of the ventricle, thereby imitating the native movements of the apex. Conceptually, the system facilitates ventricular torsion mechanics and physiologic blood flow dynamics: the stress is transposed from the weakened myocardial segments to the nitinol frame, generating supportive work in diastolic filling due to its flexible physical characteristics. In this regard, the device can hypothetically contribute to the twisting-untwisting mechanics and to the reduction of the intraventricular wall stress, improving overall myocardial efficiency. Animal data support this concept, demonstrating a leftward shift in the pressure-volume relationships [1].

Clinical experience has been gathered in various registries and single center experience $[2,3]$ as well as
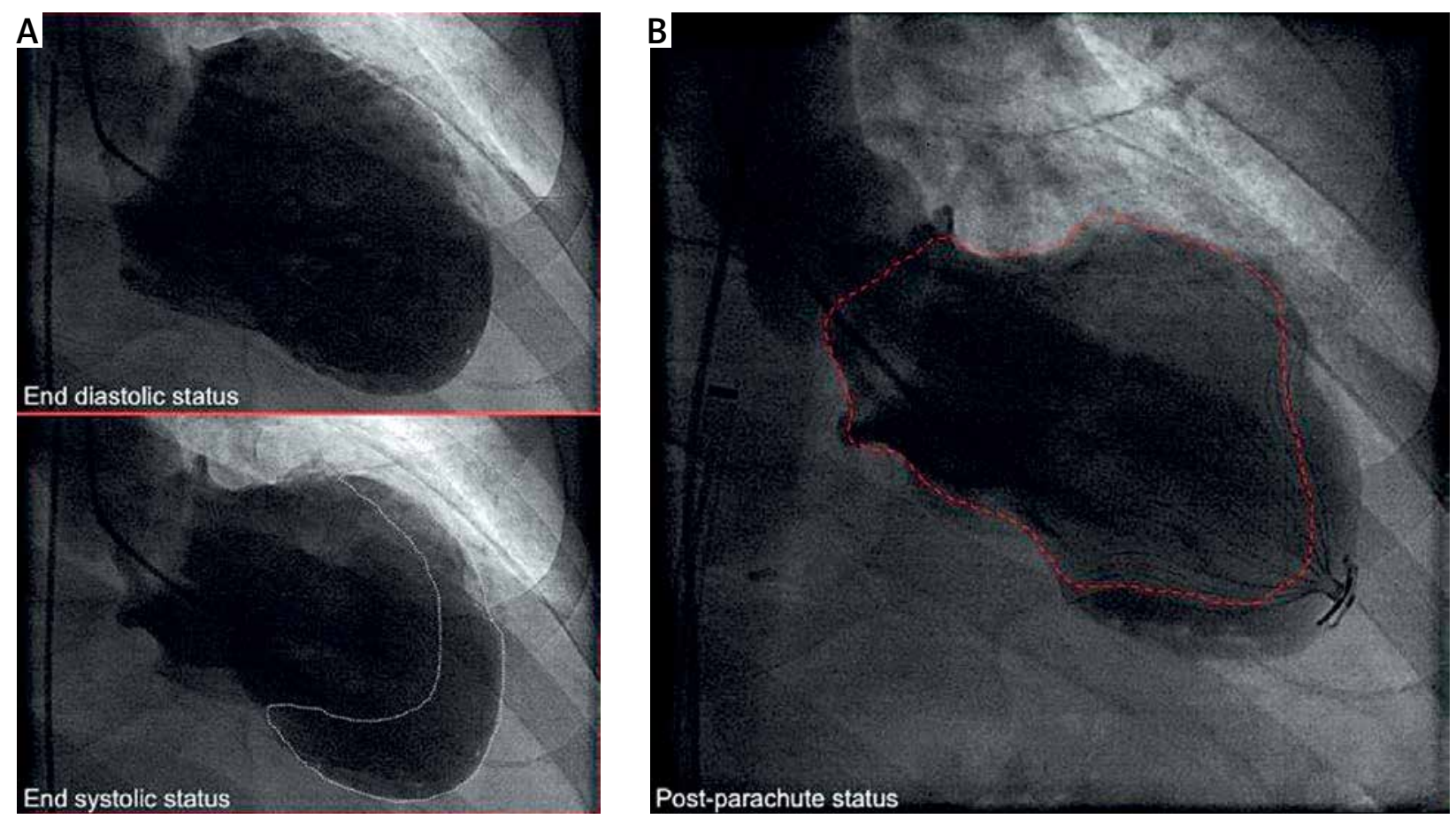

Figure 1. Remodeled left ventricle after extensive anterior myocardial infarction can be functionally divided into a dynamic and a static space (indicated in Panel A - lower picture). The Parachute device (CardioKinetix Inc., Menlo Park, CA, USA) aims to partition distal dysfunctional segments that are non-contributory to the ventricular mechanics and forward cardiac output and exclude them from the blood circulation in order to restore the near-physiologic shape and structure of the left ventricle (indicated in Panel B) 
the initial PARACHUTE trial [4]. Clinical experience so far indicates an overall favorable safety profile with several signs of improvement as regards the clinical symptoms, cardiac structure and function and physical fitness. Our personal perspective and experience with this innovative approach to modify left ventricular geometry also confirm a favorable safety profile. The key is appropriate patient selection in the subgroup of symptomatic patients with chronic heart failure after extensive anterior myocardial infarction and relevant apical scar. The pre-procedural selection and planning are aided by computed tomography or magnetic resonance imaging, determining the anatomic suitability for the intervention. The procedure requires smooth peripheral vascular access. Care should be paid to the aortic valve crossing, which has been recently facilitated by improvements in guide design and sizing. The implantation itself should aim at restoration of the near-physiologic geometry. Therefore, taking into account the pre-procedural evaluation, accurate 3-dimensional thinking is required for proper positioning of the device. Various sizes are available, expanding the patients' eligibility and anatomic substrate. Postprocedural systemic anticoagulation is required at least for one year, but the need for longer anticoagulation treatment is unclear. Further randomized studies are ongoing to establish its clinical value in treatment of post-infarct heart failure [5].

\section{Left atrial depressurization}

Diastolic heart failure or heart failure with preserved left ventricular ejection fraction is characterized by abnormal relaxation and chamber stiffness. The main therapeutic goal achieved by either medical or device-based interventions should be to enhance diastolic relaxation and reduce myocardial stiffness in tandem with the reduction of afterload and diastolic pressure load.
A

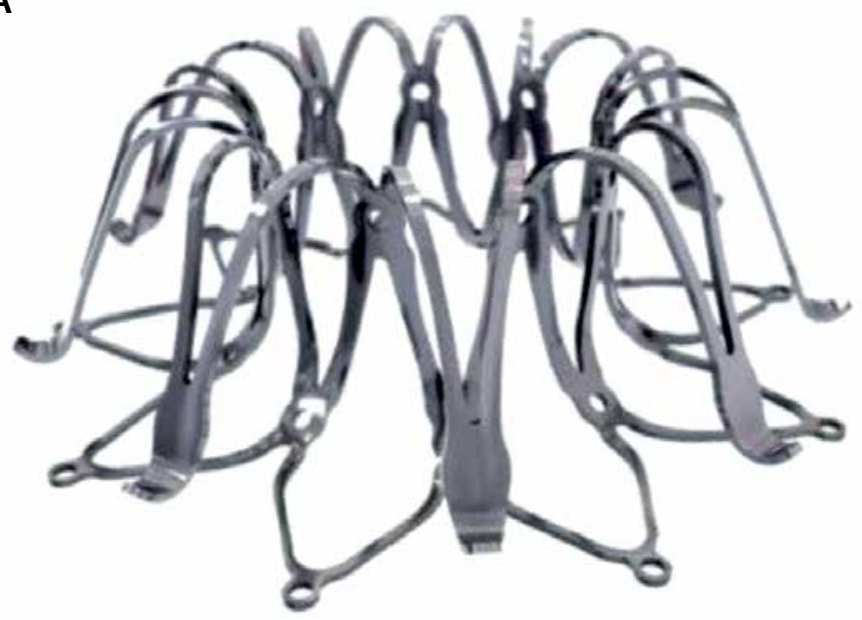

B
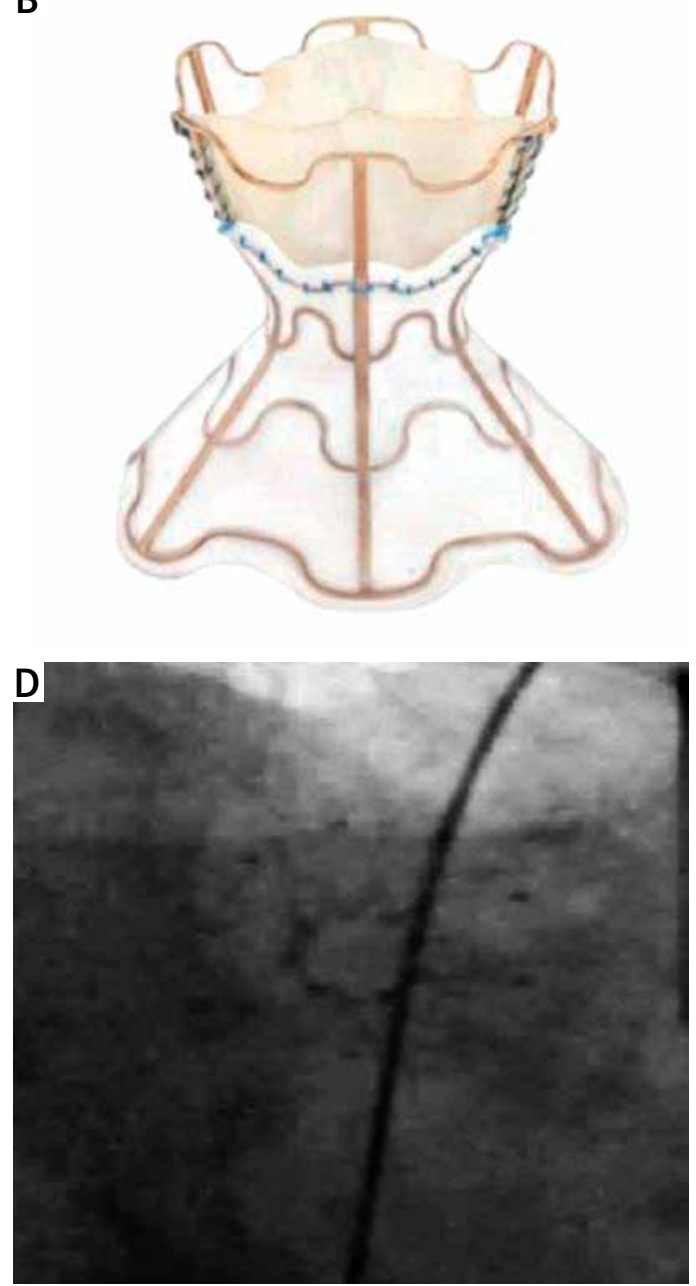

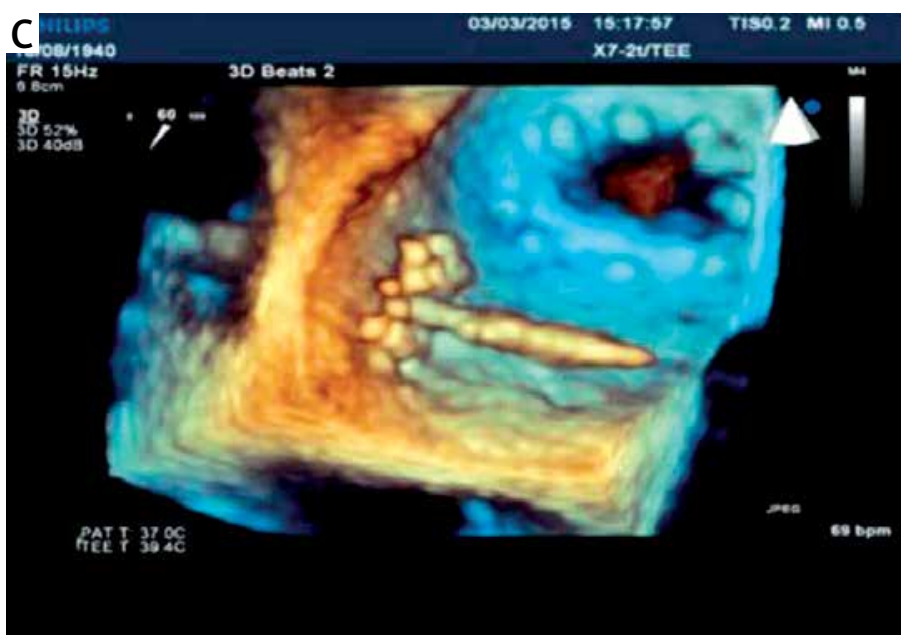

Figure 2. A, B - IASD (Corvia Medical Inc., Tewksbury, MA, USA) (A) and V-Wave Shunt (V-Wave Ltd, Or Akiva, Israel) (B). Both are designed to create a controlled atrial septal defect in symptomatic patients with heart failure. C, D - 3D echocardiographic appearance of the IASD device after deployment in the left atrium (C) and final fluoroscopic appearance of the device after the complete deployment implantation (D) 
Elevated left atrial pressures and pulmonary congestion related to backward failure are among the hallmarks of severe heart failure with preserved left ventricular ejection fraction, and they are considered to be the main source of symptoms and reduced exercise tolerance [6, 7]. Since there is no causative therapy to treat diastolic heart failure, current strategies focus on the treatment of modifying factors and comorbidities and use of diuretics. Recently, new catheter-based approaches were proposed to reduce left atrial pressure and ventricular decompression: the IASD (Corvia Medical Inc., Tewksbury, MA, USA) (Figure $2 \mathrm{~A}$ ) and the V-Wave Shunt (V-Wave Ltd, Or Akiva, Israel) (Figure 2 B). Both are designed to create a controlled atrial septal defect in symptomatic patients with heart failure. While the W-Wave Shunt device is being tested in both systolic and diastolic heart failure, the IASD device is being evaluated in patients with symptomatic heart failure and ejection fraction $\geq 40 \%$. The feasibility and early safety profile of both device-based approaches have been demonstrated in the initial studies [8,9]. The authors have personal experience with use of the IASD device (within the context of the REDUCE Elevated Left Atrial Pressure registry with the primary goal to evaluate safety and performance of the device implant) [10]. The device is percutaneously implantable through conventional venous access and transseptal puncture. The selection in the registry has been guided by the clinical and hemodynamic criteria, including levels of LV filling pressures at rest or during supine exercise. The procedure itself is straightforward. After trans-septal puncture, the preloaded device is advanced to the left atrial site of the septum. Then, the left atrial side of the device is released under echocardiographic and fluoroscopic guidance followed by slight retraction of the handle and release of the right atrial side. The postprocedural regimen includes aspirin 75-325 mg. Concomitant use of other antiplatelet or anticoagulant regimens is prescribed per institutional practice or individual patient profile. The first outcome data are expected to be presented at the upcoming ACC 2016 meeting.

\section{Global clinical perspective of catheter-based interventions in heart failure}

Considering the profound mechanism of heart failure, namely impaired myocardial function at the cellular, tissue and system level, there is no question that medical therapy targeting symptoms and deleterious compensatory mechanisms remains the mainstay in state-of-the art management of heart failure. Cardiac resynchronization therapy is the first example of successful device-based interventions altering the adverse course of heart failure. Its success as well as detailed knowledge of factors and mechanisms contributing to heart failure progression facilitated the development of device-based interventions for treatment of chronic heart failure. While the assist devices are aimed at end-stage heart failure, emerging device-based percutaneous or minimal invasive techniques comprise a wide spectrum of innovative concepts that target ventricular remodeling, cardiac contractility or neuro-humoral modulation. The clinical adoption is in the early stages of the initial feasibility and safety studies, and clinical evidence needs to be gathered in appropriately designed clinical trials.

\section{Conflict of interest}

The authors declare no conflict of interest.

\section{References}

1. Nikolic SD, Khairkhahan A, Ryu M, et al. Percutaneous implantation of an intraventricular device for the treatment of heart failure: experimental results and proof of concept. J Card Fail 2009; 15: 790-7.

2. Bozdag-Turan I, Bermaoui B, Turan RG, et al. Left ventricular partitioning device in a patient with chronic heart failure: shortterm clinical follow-up. Int J Cardiol 2013; 163: e1-3.

3. Sagic D, Otasevic P, Sievert $H$, et al. Percutaneous implantation of the left ventricular partitioning device for chronic heart failure: a pilot study with 1-year follow-up. Eur J Heart Fail 2010; 12: 600-6.

4. Mazzaferri EL Jr, Gradinac S, Sagic D, et al. Percutaneous left ventricular partitioning in patients with chronic heart failure and a prior anterior myocardial infarction: results of the PercutAneous Ventricular RestorAtion in Chronic Heart failUre PaTiEnts Trial. Am Heart J 2012; 163: 812-820.e1.

5. Costa MA, Pencina M, Nikolic S, et al. The PARACHUTE IV trial design and rationale: percutaneous ventricular restoration using the parachute device in patients with ischemic heart failure and dilated left ventricles. Am Heart J 2013; 165: 531-6.

6. Borlaug BA, Nishimura RA, Sorajja P, et al. Exercise hemodynamics enhance diagnosis of early heart failure with preserved ejection fraction. Circ Heart Fail 2010; 3: 588-95.

7. Maeder MT, Thompson BR, Brunner-La Rocca HP, Kaye DM. Hemodynamic basis of exercise limitation in patients with heart failure and normal ejection fraction. J Am Coll Cardiol 2010; 56: 855-63.

8. Amat-Santos IJ, Bergeron S, Bernier M, et al. Left atrial decompression through unidirectional left-to-right interatrial shunt for the treatment of left heart failure: first-in-man experience with the V-Wave device. Eurointervention 2015; 10: 1127-31.

9. Søndergaard L, Reddy V, Kaye D, et al. Transcatheter treatment of heart failure with preserved or mildly reduced ejection fraction using a novel interatrial implant to lower left atrial pressure. Eur J Heart Fail 2014; 16: 796-801.

10. Hasenfuss G, Gustafsson F, Kaye D, et al. Reduce LAP-HF Trial Investigators. Rationale and design of the reduce elevated left atrial pressure in patients with heart failure (Reduce LAP-HF) trial. J Card Fail 2015; 21: 594-600. 